\title{
A Computer-Assisted Robotic Ultrasound-Guided Biopsy System for Video-Assisted Surgery
}

\author{
Giuseppe Megali ${ }^{1}$, Oliver Tonet ${ }^{1}$, Cesare Stefanini ${ }^{1}$, Mauro Boccadoro ${ }^{1}$, \\ Vassilios Papaspyropoulos ${ }^{2}$, Licinio Angelini ${ }^{2}$, and Paolo Dario ${ }^{1}$ \\ 1 MiTech Lab - Scuola Superiore Sant'Anna, Pisa, Italy \\ \{g.megali,o.tonet, c.stefanini, m. boccadoro, p. dario\}@mail-arts.sssup.it \\ 2 Dipartimento di Scienze Chirurgiche e Tecnologie Mediche Applicate, \\ Università "La Sapienza", Roma, Italy \\ \{vassilios.papas, licinio.angelini\}@uniroma1.it
}

\begin{abstract}
Current ultrasound-guided biopsy procedures used in videoassisted surgery suffer from limitations due to difficult triangulation and manual positioning of the biopsy needle. We present a prototype computer-assisted robotic system for needle positioning that operates in a synergistic way with the clinician. The performance of the system in terms of positioning accuracy and execution time is assessed. Results suggest suitability for clinical use.
\end{abstract}

\section{Introduction}

Intra-operative ultrasonography is used, in surgical practice, for many applications both for diagnostic and therapeutic purposes [1. Thanks to its specificity and sensitivity, it is of primary importance in disorders of the pancreas [2], biliary tree [3, and liver 4, especially in early stages of the disease.

The diagnostic accuracy of alternative techinques, i.e. pre-operative imaging (angiography, scintigraphy, CT, ultrasonography), bio-humoral surveys, and surgical exploration, does not exceed $60-80 \%[5]$. On the other hand, intra-operative ultrasonography allows early diagnosis and precise localization - and thus accurate and radical surgical treatment - of lesions that are not otherwise detectable.

As intra-operative ultrasonography provides real-time data, it can be applied on demand to assist the surgeon during intervention. A major diagnostic application is providing guidance for percutaneous and endoscopic biopsy.

In current clinical practice, percutaneous ultrasound-guided biopsy is performed either freehand or by means of a biopsy kit, consisting of an ultrasound (US) probe equipped with a cylindrical needle guide, hinged at a fixed distance to a beam integral with the probe. This contrivance forces the needle to stay in the imaging plane of the probe, so that the needle tip is always visible in the US image, contrary to what happens in freehand conditions. The hinge on the needle guide allows to vary the needle insertion angle. 
The main drawback of this technique is that, since the needle is anchored to the probe, a difficult compromise is necessary between the probe position that provides the best images and the most convenient insertion point for biopsy.

Small US transducers, mounted in probes of about $1 \mathrm{~cm}$ diameter, are of particular interest in minimally invasive surgery. These probes can be introduced directly into the patient's body to obtain better quality images of the internal organs. Anyway, whenever the bioptic procedure cannot be carried out percutaneously, as in the case of video-assisted (laparoscopic or thoracoscopic) interventions, no biopsy kits are available and only freehand approach is possible.

Several systems have been proposed for providing assistance to needle positioning, using ultrasonography [6], or fluoroscopy [7] 8].

In this paper we present a prototype robotic tool [9] for US-guided biopsy during video-assisted surgery, that operates in a synergistic way with the clinician. The system allows precise 3D localization and visualization of the biopsy target without the need for pre-operative imaging, contrast agents, or intraoperative X-rays. The system is modular and was conceived as feasibility study, for evaluation of the overall performance and suitability for clinical use.

\section{System Overview}

\subsection{A More Accurate Biopsy Procedure}

The system, illustrated in Fig. 1] is composed of a robot arm carrying the biopsy syringe, an ultrasonography system, a 3D localizer and a PC-based main processing unit (MPU), which also implements the graphical user interface (GUI).

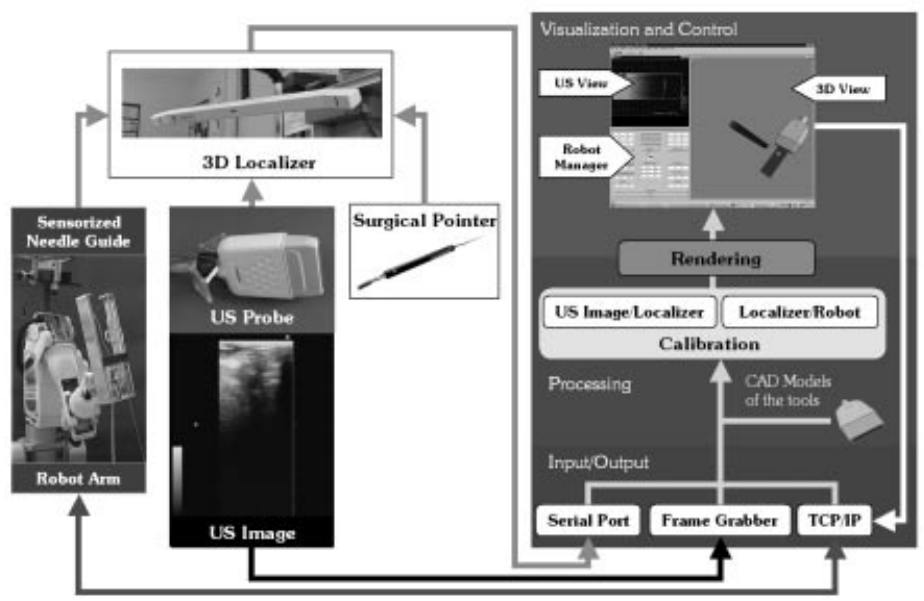

Fig. 1. System architecture and intercommunication between the modules 
The biopsy syringe is mounted on the end effector (EE) of the robot arm by means of a needle guide (Fig. 2). When the robot arm is put on place, the guide allows the needle a 1-degree of freedom (DOF) motion along its axis.

The system is designed to provide great accuracy, while keeping the biopsy procedure simple and intuitive: the clinician selects the biopsy target directly on the US image and the insertion point on the patient's body. Subsequently, the robot arm positions the biopsy needle along the defined trajectory. In order to guarantee maximum safety of the procedure, the insertion of the needle and the bioptic sampling is left to the manual execution of the clinician.

From the end user's viewpoint, the procedure consists of the following steps:

target identification: the clinician scans the region of interest with the US probe in order to locate the biopsy target;

target selection: the clinician selects the biopsy target point on the US image shown in the GUI, by clicking on it with the mouse pointer;

insertion point selection: the clinician uses a surgical pointer, connected to the localizer, to select the insertion point for the biopsy needle;

robot positioning: the robot places the EE on the resulting trajectory, so that the needle will reach, at the end of stroke of the guide, the biopsy target;

stop of breath: to avoid the natural movement of the parenchymal structures during respiratory cycle, we considered the anaesthesiological interruption of the patient's breath for the final steps of the procedure;

robot position adjustment: using the US probe and the GUI, the clinician evaluates the accuracy of the needle positioning. If necessary he/she selects the target point again and commands an adjustment of the robot position; bioptic sampling: the clinician releases the slide which carries the needle, manually slides it down until the end of stroke, and executes the sampling.

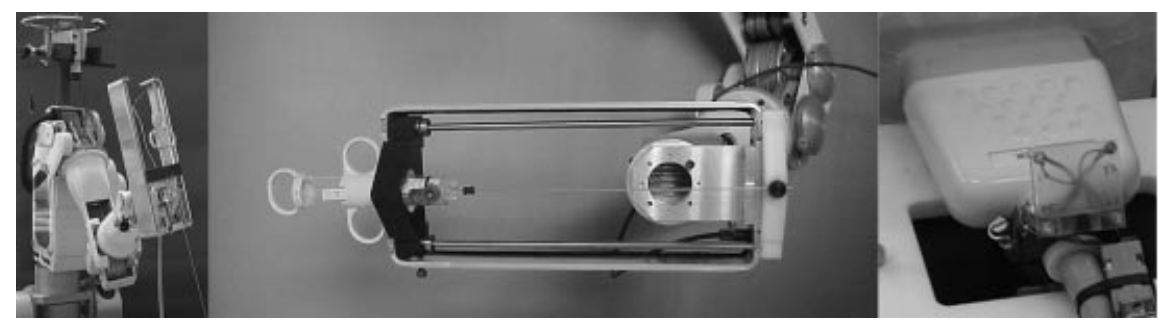

Fig. 2. Hardware components of the system: (a) Dexter arm carrying the (b) sensorized needle guide; (c) sensorized US probe

\subsection{Description of the System Components}

The robot is an 8-DOF Dexter arm 1. Whereas it is not designed for surgery applications, its workspace is suitable for this prototype implementation.

\footnotetext{
${ }^{1}$ Dexter arm, S.M. Scienzia Machinale srl, Pisa, Italy.
} 
The ultrasonography system is a portable system 2 equipped with a $7.5 \mathrm{MHz}$ linear probe. In the oncoming clinical trials the system will be replaced by a laparoscopic ultrasonography system with an articulated, sensorized probe.

The localizer is an optical system 3 , consisting of three arrays of cameras that detect the pulsed light, emitted by small infrared LEDs, placed on the objects to track: the EE of the robot, the US probe and the surgical pointer.

The position of the objects tracked by the localizer are transmitted to the MPU4 through a serial port. Bidirectional communication between the MPU and the Dexter arm is established via TCP/IP ethernet. The MPU digitizes and displays the analog video image exported by the ultrasonography system by means of a frame grabber.

The following subsections give some deeper insight on the main custom-made components of the system: the sensorized needle guide and the GUI.

Sensorized Needle Guide This component has to provide a linear motion to the biopsy syringe during insertion. It is positioned at the robot's EE level (see Fig. 2), and is sensorized with four LEDs located in proximity of the guide corners, since large distances allow higher localization accuracy.

The mechanical structure has to fit three requirements: lightness, precision, and smoothness of operation. Lightweight aluminum alloy was used to fabricate the mechanical frame. Linear bearings allow smooth operation. In order to increase the bending stiffness of the system, their shafts are fixed in the seats. The overall weight of the guide is $0.18 \mathrm{~kg}$, widely below the robot payload.

The Graphical User Interface The GUI is displayed on the screen of the MPU and is designed to allow an intuitive visual selection of the biopsy target point, an easier orientation of the probe with respect to the target point, to control the robot movements and evaluate the accuracy of robot positioning. The GUI comprises three windows, as illustrated in Fig. 3:

US view: this window shows the US image captured by the frame grabber. In order to plan the trajectory of needle insertion, a marker, updated in realtime, is displayed on the intersection point between the trajectory of the needle and the US image plane;

3D view: this window shows a virtual scenario in which the relative positions of the CAD models of the sensorized tools are updated in real-time. The target and insertion points, selected by the clinician, are also marked in the scene, simplifying the positioning of the tools relative to them, e.g. for refinding the biopsy target on the US image after having moved the probe;

robot manager: this window contains the "move robot" button and shows all parameters used for robot control. In the final version there will be no

\footnotetext{
${ }^{2}$ HS-1201 Linear Scanner, Honda Electronics Co., LTD., Toyohashi, Aichi, Japan.

${ }^{3}$ FlashPoint ${ }^{\circledR} 5000$ 3D Localizer, Image Guided Technologies, Inc., Boulder, CO, USA.

4 TDZ 2000 GX1 workstation, Intergraph Corp., Huntsville, AL, USA.
} 
need for displaying parameters and the clinician will use a pedal to send commands to the robot.

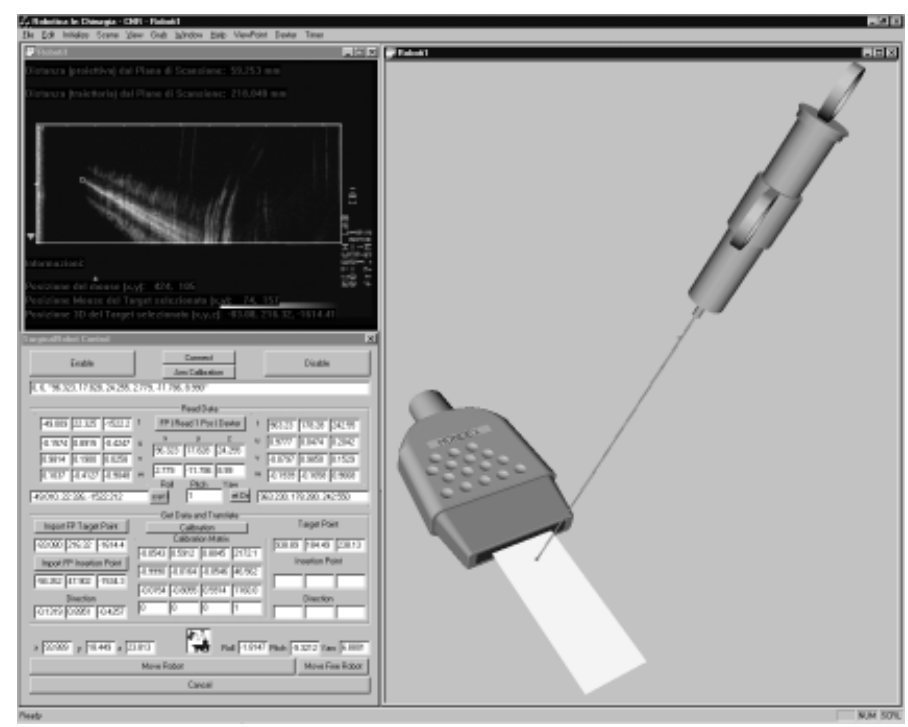

Fig. 3. A screenshot of the graphical user interface, containing the US view (upper left), the 3D view (right) and the robot manager window (lower left)

\section{Methods}

The biopsy procedure is now illustrated from a methodological point of view:

localization of target point: the user selects the biopsy target point on the US view, with a mouse click. Since the window that displays the image has been calibrated, the pixel coordinates of the chosen point are converted in metric coordinates, referred to the US probe position. The position of the US probe is given in the localizer reference frame (LRF), therefore computation of the $3 \mathrm{D}$ position of the biopsy target is straightforward;

localization of insertion point: LRF coordinates of the point on the patient's skin, selected by means of the sensorized surgical pointer, are measured directly by the $3 \mathrm{D}$ localizer;

computation of needle guide positioning: the trajectory of needle insertion is the straight line through the target point and the insertion point. The robot EE is positioned so that the needle tip, at end of stroke of the slide, reaches the target point. These requirements lock 5 of the 6 DOF of the EE. The residual $1 \mathrm{DOF}$, i.e. the rotation of the slide around the needle axis, is chosen so to maximize visibility of the needle guide to the localizer; 
hand-eye calibration: selection of insertion and target points is done in LRF coordinates. To position the robot arm, computation of the corresponding points in the robot reference frame $(\mathrm{RRF})$ is required. This operation, called hand-eye calibration, requires the simultaneous measurement of at least three non-colinear points in the two reference frames. By considering relative movements, the problem can be cast in the form $A X=X B$, where $A, B$, and $X$ are $4 \times 4$ transformation matrices describing the position of the needle guide in the LRF, the position of the robot end-effector in the RRF, and the transformation between the two reference frames, respectively. A leastsquares solution for $X$ can be found in [10];

robot positioning: due to the calibration inaccuracy, the positioning of the EE is affected by an error, increasing with the magnitude of robot movement. In order to minimize the amplification of the error due to large movements, the positioning of the robot involves two phases: first, the robot performs a coarse positioning of the needle guide on the planned trajectory; second, a fine positioning movement corrects the mismatch, measured in the LRF, between the planned and reached trajectory. This phase, supervised by the operator, can be iterated to obtain a very accurate positioning, thus implementing an interactive closed loop for accuracy control;

needle insertion and sampling: performed manually by the clinician.

\section{Measurement of the Performance}

To evaluate the suitability of the prototype system for clinical use, system performance has been assessed in terms of accuracy and execution time.

Measurements are conducted under ideal conditions (see Fig. 4): the biopsy target, a fixed and pointed wooden stick, was immersed in water, so to avoid image distortions due to inhomogeneous medium, and needle deformations induced by contact with solid tissues surrounding the target. To minimize inaccuracy due to the US image thickness, the biopsy target was placed on the center plane of the US beam.

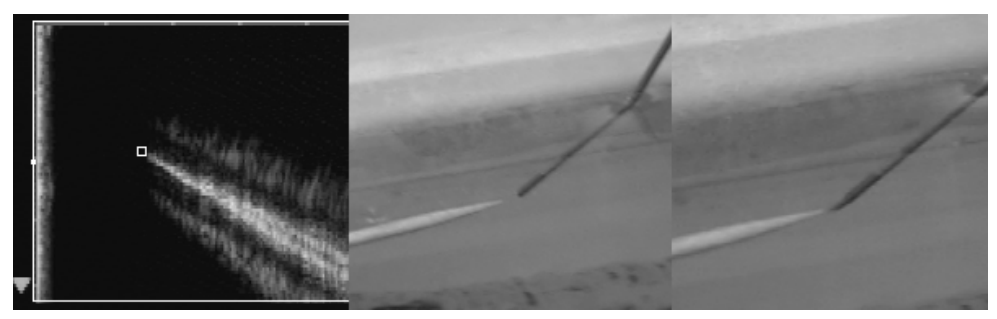

Fig. 4. US View highlighting the biopsy target point and the needle positions reached after one and two fine positioning cycles 
The accuracy of the system has been assessed by measuring, on 30 different target points distributed in the surgical workspace, the deviation of the needle tip from the target point.

The results are presented in the following table, where $\varepsilon_{1}, \varepsilon_{2}$, and $\varepsilon_{3}$ are the deviations, measured by the localizer, respectively after one, two, and three cycles of fine robot positioning, while $\varepsilon_{\mathrm{US}}$ is the deviation, measured on the US image, between the wooden point and the needle tip, after the third cycle:

\begin{tabular}{|l|c|c|c|c|}
\hline & $\varepsilon_{1}$ & $\varepsilon_{2}$ & $\varepsilon_{3}$ & $\varepsilon_{\text {US }}$ \\
\hline Mean error & $1.32 \mathrm{~mm}$ & $0.85 \mathrm{~mm}$ & $0.63 \mathrm{~mm}$ & $2.05 \mathrm{~mm}$ \\
\hline Max error & $2.13 \mathrm{~mm}$ & $1.39 \mathrm{~mm}$ & $0.88 \mathrm{~mm}$ & $2.49 \mathrm{~mm}$ \\
\hline
\end{tabular}

The results obtained for the variables $\varepsilon_{1,2,3}$ show that the system achieves sub-millimetric accuracy in the positioning of the needle guide after two fine positioning cycles of the robot. In other words, it is possible to compensate for the inaccuracy of the robot arm (about $2 \mathrm{~mm}$ ) by means of fine positioning movements; the overall accuracy of the system depends on the accuracy of the localizer [11, on the manufacturing of the frames holding the LEDs, and on calibration and thickness of the US images.

While the error measured by $\varepsilon_{i}$ is relative to the needle guide positioning, $\varepsilon_{\mathrm{US}}$ is the error measured at the needle tip, when the slide is at end of stroke. The difference between the two is mostly due to the deflection of the needle.

Measurements on time consumption show that the whole procedure, excluding the time spent by the clinician for ultrasonography exploration, requires about $1 \mathrm{~min}$. The breath of the patient has to be stopped only during fine positioning of the robot (i.e. about $15 \mathrm{~s}$ for each cycle) and the sampling phase.

\section{Conclusions}

We presented a prototype robotic US-guided system for video-assisted surgery. The system improves the current US-guided biopsy procedures by offering intuitive selection of the biopsy target point and automated, accurate positioning of the biopsy needle. Featuring needle trajectory locking, the system allows to safely reach the target with a single puncture; this cannot be guaranteed in freehand approach. Experimental results are encouraging and compatible with clinical requirements, in terms of positioning accuracy and execution time.

Accuracy will be further increased by improving the overall stiffness of the robot arm and needle guide. The error due to needle deflection, $\varepsilon_{\mathrm{US}}-\varepsilon_{3}$, will be reduced by adding a second guiding support to the needle, close to the needle insertion point. Oncoming in vitro tests will assess needle deflection and US image distortion due to soft tissue surrounding the biopsy target.

Safety is a very important issue in medical robotics. The current prototype features emergency arrest and leaves to the clinician the execution of needle insertion and sampling. Furthermore, the robot, attaining the target at end of stroke of the needle guide, is kept at maximal distance from the patient. 
Future work will also focus on compliant robot control, in order to avoid interference with other devices present in the surgical scene and to prevent potential damage deriving from accidental collision.

\section{Acknowledgements}

This work has been carried out with support by the "Progetto Strategico Robotica in Chirurgia", promoted by Consiglio Nazionale delle Ricerche (CNR), Italy. The authors are grateful to Dr. M.C. Carrozza, Dr. B. Magnani, and Dr. S. D'Attanasio for their work in the early stages of the project. The authors would like to thank Dr. C. Laschi, G. Teti, and L. Zollo for providing assistance with the Dexter arm. Thanks to B. Massa and D. Rosti for the CAD drawings.

\section{References}

[1] Angelini L. and Caratozzolo M. Intraoperative echography: the state of the art. Ann Ital Chir, 70(2):223-30, March-April 1999.

[2] Galiber A.K., Reading C.C., Charboneau J.W., Sheedy P.F., James E.M., Gorman B., Grant C.S., van Heerden J.A., and Telander R.L. Localization of pancreatic insulinoma: comparison of pre- and intraoperative US with CT and angiography. Radiology, 166(2):405-8, February 1988.

[3] Orda R., Sayfan J., Strauss S., Barr J., and Oland J. Intra-operative ultrasonography as a routine screening procedure in biliary surgery. Hepatogastroenterology, 41(1):61-64, February 1994.

[4] Castaing D., Emond J., Kunstlinger F., and Bismuth H. Utility of operative ultrasound in the surgical management of liver tumors. Ann Surg, 204(5):600-5, 1986.

[5] Russo A., La Rosa C., Cajozzo M., Spallitta I., Demma I., Modica G., and Bazan P. Screening for liver metastases of colorectal carcinoma by the routine use of intraoperative echography. Minerva Chir, 17(44):1893-900, 1989.

[6] O. Chavanon, C. Barbe, J. Troccaz, and L. Carrat. Computer-ASsisted PERicardial punctures: animal feasibility study. Proc. CVRMed-MRCAS'97, Lecture Notes in Computer Science, 1205:285-94, March 1997.

[7] Dan Stoianovici, Louis L. Whitcomb, James H. Anderson, Russell H. Taylor, and Louis R. Kavoussi. A modular surgical robotic system for image guided percutaneous procedures. Proc. MICCAI'98, Lecture Notes in Computer Science, 1496:404-410, October 1998.

[8] A. Bzostek, S. Schreiner, A. C. Barnes, and J. A. Cadeddu. An automated system for precise percutaneous access of the renal collecting system. Proc. CVRMedMRCAS'97, Lecture Notes in Computer Science, 1205:299-308, March 1997.

[9] P. Dario, M.C. Carrozza, M. Marcacci, S. D'Attanasio, B. Magnani, O. Tonet, and G. Megali. A novel mechatronic tool for computer-assisted arthroscopy. IEEE Transactions on Information Technology in Biomedicine, 4(1):15-29, March 2000.

[10] F.C. Park and B.J. Martin. Robot Sensor Calibration - Solving $A X=X B$ on the Euclidean Group. IEEE Trans. Robotics and Automation, 10(5):717-721, 1994.

[11] F. Chassat and S. Lavallée. Experimental protocol of accuracy evaluation of 6-d localizers for computer-integrated surgery. application to four optical localizers. Proc. MICCAI'98, Lecture Notes in Computer Science, 1496:277-284, October 1998. 